\title{
When somebody loses weight, where does the fat go?
}

In figure 2 of this Christmas Feature by Ruben Meerman and Andrew Brown (BMJ 2014;349:g7257, doi:10.1136/bmj.g7257) the number in pale blue above $\mathrm{H}_{2} 0$ should be $9.4 \mathrm{~kg}$, not " 8.4 $\mathrm{kg}$ " as was published. This error in figure 2 was corrected online on 3 February 2015
Cite this as: BMJ 2014;349:g7782

๑ BMJ Publishing Group Ltd 2014 